\title{
FUNCTORIAL FINITE SUBCATEGORIES OVER TRIANGULAR MATRIX RINGS
}

\author{
S. O. SMALØ
}

(Communicated by Maurice Auslander)

\begin{abstract}
Let $\Lambda$ and $\Gamma$ be Artin algebras, $M$ a $\Gamma$ - $\Lambda$-bimodule, and $R$ the triangular matrix ring of $\Lambda, \Gamma$, and $M$; assume that $R$ is also an Artin algebra. The $R$-modules are triples $(U, V, f)$ where $U$ is a $\Lambda$-module, $V$ is a $\Gamma$-module, and $f$ is a $\Gamma$-homomorphism from $M \otimes U$ to $V$. For an Artin algebra $S$, let $\bmod S$ denote the category of finitely generated $S$-modules. For full subcategories $\mathscr{S}$ of $\bmod \Lambda$ and $\mathscr{T}$ of $\bmod \Gamma$, let $\bmod R_{\mathscr{S}}^{\mathscr{S}}$ denote the full subcategory consisting of the modules $(U, V, f)$, where $U$ is in $\mathscr{S}$ and $V$ is in $\mathscr{T}$. In this paper it is proved that $\bmod R_{\mathscr{S}}^{\mathscr{S}}$ is functorially finite in $\bmod R$ if and only if $\mathscr{S}$ is functorially finite in $\bmod \Lambda$ and $\mathscr{T}$ is functorially finite in $\bmod \Gamma$. Using this result, we increase the known examples of functorially finite subcategories considerably, hence also the classes of subcategories having relative almost split sequences.
\end{abstract}

\section{INTRODUCTION}

In studying the category of representations of a partially ordered set, the category of lattices over an order, or other similar structures, one often gets involved in studying full subcategories of the category of modules over an Artin algebra. Usually these subcategories are additive, with splitting idempotents satisfying some additional hypothesis, such as being closed with respect to extensions. Therefore the questions of existence of almost split sequences arise naturally in connection with the presentation of both the simple contravariant and covariant functors. In this connection the notion of functorial finiteness from [AS1] is of importance.

The aim of this note is to show that the functorial finiteness of subcategories is preserved under some triangular matrix ring constructions. It can also be generalized to the setting of comma categories. Using this result for Artin algebras, the existence of almost split sequences for the category of prinjective modules studied by Grecht [Gr] and de la Peña and Simson [PS] follows immediately. This result will increase considerably the number of known examples of other types of subcategories having almost split sequences.

Received by the editors November 21, 1989.

1980 Mathematics Subject Classification (1985 Revision). Primary 16A64, 16A46; Secondary $18 \mathrm{~A} 25$. 


\section{Preliminaries}

In this section we recall the definitions and notions needed in the rest of the paper and fix the notation.

For a ring $S$, we let $\bmod S$ denote the category of finitely presented left $S$-modules. Let $\Lambda$ and $\Gamma$ be Artin $T$-algebras, where $T$ is a commutative Artin ring, and let ${ }_{\Gamma} M_{\Lambda}$ be a $\Gamma$ - $\Lambda$-bimodule, where the induced actions of $T$ through $\Lambda$ and $\Gamma$ commutes and $M$ is finitely generated as a $T$-module. Then form the triangular matrix ring $R$ given by

$$
R=\left\{\left(\begin{array}{cc}
\lambda & 0 \\
m & \gamma
\end{array}\right) \mid \lambda \in \Lambda, m \in{ }_{\Gamma} M_{\Lambda}, \text { and } \gamma \in \Gamma\right\},
$$

where the operations are the usual matrix operations. It is clear that $R$ is also an Artin $T$-algebra. A left $R$-module may be viewed as a triple $(U, V, f)$, where $U$ is a $\Lambda$-module, $V$ is a $\Gamma$-module, and $f$ is a $\Gamma$-homomorphism from ${ }_{\Gamma} M_{\Lambda} \otimes U$ to $V$. As an Abelian group, $(U, V, f)$ is just $U \times V$, and the multiplication with an element from $R$ is given by

$$
\left(\begin{array}{cc}
\lambda & 0 \\
m & \gamma
\end{array}\right)(u, v)=(\lambda u, m f(u)+\gamma v),
$$

for $\left(\begin{array}{ll}\lambda & 0 \\ m & \gamma\end{array}\right) \in R$ and $(u, v) \in U \times V$. An $R$-homomorphism $\phi:(U, V, f) \rightarrow$ $\left(U^{\prime}, V^{\prime}, f^{\prime}\right)$ is a pair $(\alpha, \beta)$, where $\alpha: U \rightarrow U^{\prime}$ is a $\Lambda$-homomorphism and $\beta: V \rightarrow V^{\prime}$ is a $\Gamma$-homomorphism such that $f^{\prime}(M \otimes \alpha)=\beta f$. An alternative description can be obtained by using the adjoint isomorphism

$$
\operatorname{Hom}_{\Gamma}\left({ }_{\Gamma} M_{\Lambda} \otimes U, V\right) \simeq \operatorname{Hom}_{\Lambda}\left(U, \operatorname{Hom}_{\Gamma}\left(\Gamma_{\Gamma} M_{\Lambda}, V\right)\right) .
$$

A description of the left $R$-modules is then obtained as a triple $(U, V, f)$, where $U$ is a $\Lambda$-module, $V$ is a $\Gamma$-module, and $f$ is a $\Lambda$-homomorphism from $U$ to $\operatorname{Hom}_{\Gamma}\left({ }_{\Gamma} M_{\Lambda}, V\right)$. As an Abelian group, $(U, V, f)$ is as before, but the multiplication with an element from $R$ is now given by

$$
\left(\begin{array}{cc}
\lambda & 0 \\
m & \gamma
\end{array}\right)(u, v)=(\lambda u, f(u)(m)+\gamma v) \text {. }
$$

It is left to the reader to describe the $R$-homomorphisms in this case.

We now recall the notion of covariantly, contravariantly, and functorially finite subcategories from [AS1].

Recall that a covariant functor $F$ from a category $\mathscr{A}$ to the category of sets is said to be finitely generated if there is an object $A$ in $\mathscr{A}$ and a natural transformation from $\mathscr{A}(A$,$) onto F$. The definition of a finitely generated contravariant functor is analogous.

Definition. A full subcategory $\mathscr{A}$ of a category $\mathscr{B}$ is said to be

(i) contravariantly finite in $\mathscr{B}$ if, for each object $X$ in $\mathscr{B}$, the representable functor $\mathscr{B}(, X)$ restricted to $\mathscr{A}$ is finitely generated as a functor on $\mathscr{A}$, 
(ii) covariantly finite in $\mathscr{B}$ if, for each object $X$ in $\mathscr{B}$, the representable functor $\mathscr{\mathscr { R }}(X$,$) restricted to \mathscr{A}$ is finitely generated, and

(iii) functorially finite in $\mathscr{B}$ if it is both contravariantly and covariantly finite in $\mathscr{B}$.

\section{THE RESULT}

Let $\Gamma, \Lambda,{ }_{\Gamma} M_{\Lambda}$, and $R$ be as before, and let $\mathscr{S}$ be a full subcategory of $\bmod \Lambda$ and $\mathscr{T}$ be a full subcategory of $\bmod \Gamma$. Let $\bmod R_{\mathscr{T}}^{\mathscr{S}}$ denote the full subcategory of $\bmod R$ consisting of the modules of the form $(U, V, f)$ where $U$ is in $\mathscr{S}$ and $V$ is in $\mathscr{T}$. With this notation we have the following result:

Theorem 2.1. (i) The subcategory $\bmod R_{\mathscr{G}}^{\mathscr{S}}$ of $\bmod R$ is contravariantly finite in $\bmod R$ if and only if $\mathscr{S}$ is contravariantly finite in $\bmod \Lambda$ and $\mathscr{T}$ is contravariantly finite in $\bmod \Gamma$.

(ii) The subcategory $\bmod R_{\mathscr{S}}^{\mathscr{S}}$ of $\bmod R$ is covaraintly finite in $\bmod R$ if and only if $\mathscr{S}$ is covariantly finite in $\bmod \Lambda$ and $\mathscr{T}$ is covariantly finite in $\bmod \Gamma$.

(iii) The subcategory $\bmod R_{\mathscr{G}}^{\mathscr{S}}$ of $\bmod R$ is functorially finite in $\bmod R$ if and only if $\mathscr{S}$ is functorially finite in $\bmod \Lambda$ and $\mathscr{T}$ is functorially finite in $\bmod \Gamma$.

Proof. We prove (i) and leave the rest to the reader since the proofs of (i) and (ii) are dual of each other and (iii) follows from (i) and (ii).

We first establish the "only if" part of (i). Assume $\bmod R_{\mathscr{T}}^{\mathscr{S}}$ is contravariantly finite in $\bmod R$, and let $X$ be a module in $\bmod \Lambda$. Consider the module $(X, 0,0)$ in $\bmod R$. By assumption, there exists a module $(U, V, f)$ in $\bmod R_{\mathscr{G}}^{\mathscr{S}}$ and a homomorphism $\phi=(\alpha, \beta):(U, V, f) \rightarrow(X, 0,0)$ such that each homomorphism $\psi$ from an object $\left(U^{\prime}, V^{\prime}, f^{\prime}\right)$ in $\bmod R_{\mathscr{G}}^{\mathscr{S}}$ to $(X, 0,0)$, factors through $\phi$; this will hold especially for each module of the form $\left(U^{\prime}, 0,0\right)$ in $\bmod R_{\mathscr{S}}^{\mathscr{S}}$. But then we get that $\operatorname{Hom}_{\Lambda}(, \alpha): \operatorname{Hom}_{\Lambda}(, U) \rightarrow$ $\operatorname{Hom}_{\Lambda}(, X)$ restricted to $\mathscr{S}$ is surjective. This shows that $\mathscr{S}$ is contravariantly finite in $\bmod \Lambda$. The proof that $\mathscr{T}$ is contravariantly finite in $\bmod \Gamma$ when $\bmod R_{\mathscr{G}}^{\mathscr{S}}$ is contravariantly finite in $\bmod R$ is as simple and therefore left to the reader.

Assume now that $\mathscr{S}$ is contravariantly finite in $\bmod \Lambda$ and that $\mathscr{T}$ is contravariantly finite in $\bmod \Gamma$. Let $(X, Y, g)$ be a module in $\bmod R$. By assumption, there exists a $Y_{\mathscr{G}}$ in $\mathscr{T}$ and a $\Gamma$-homomorphism $t_{Y}: Y_{\mathscr{T}} \rightarrow Y$ such that

$$
\left.\left.\operatorname{Hom}_{\Gamma}\left(, Y_{\mathscr{G}}\right)\right|_{\mathscr{g}} \stackrel{\operatorname{Hom}_{\Gamma}\left(, t_{Y}\right)}{\longrightarrow} \operatorname{Hom}_{\Gamma}(, Y)\right|_{\mathscr{F}} \rightarrow 0
$$


is exact, where $\left.\right|_{\mathscr{G}}$ means restriction to $\mathscr{T}$. Now consider the pullback diagram

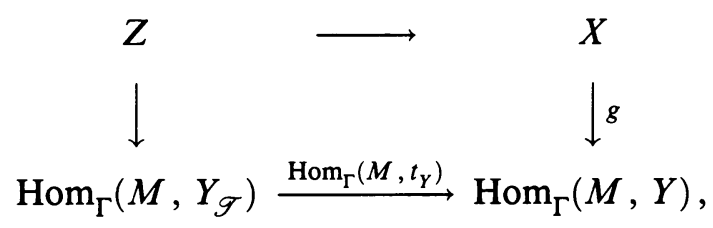

in $\bmod \Lambda$. From the assumption that $\mathscr{S}$ is contravariantly finite in $\bmod \Lambda$, it follows that there exists a $Z_{\mathscr{S}}$ in $\mathscr{S}$ and a $\Lambda$-homomorphism $s_{Z}: Z_{\mathscr{S}} \rightarrow Z$ such that

$$
\left.\left.\operatorname{Hom}_{\Lambda}\left(, Z_{\mathscr{S}}\right)\right|_{\mathscr{S}} \stackrel{\operatorname{Hom}_{\Lambda}\left(, s_{Z}\right)}{\rightarrow} \operatorname{Hom}_{\Lambda}(, Z)\right|_{\mathscr{S}} \rightarrow 0
$$

is exact. We then obtain the module $\left(Z_{\mathscr{S}}, Y_{\mathscr{T}}, h\right)$ in $\bmod R_{\mathscr{S}}^{\mathscr{S}}$, where $h$ is the composed homomorphism $Z_{\mathscr{S}} \stackrel{s_{Z}}{\rightarrow} Z \rightarrow \operatorname{Hom}_{\Gamma}\left(M, Y_{\mathscr{G}}\right)$, and we also have an $R$-homomorphism from $\left(Z_{\mathscr{S}}, Y_{\mathscr{g}}, h\right)$ to $(X, Y, g)$. The claim is that this $R$-homomorphism $\left(Z_{\mathscr{S}}, Y_{\mathscr{T}}, h\right) \rightarrow(X, Y, g)$ has the property that the induced sequence

$$
\left.\left.\operatorname{Hom}_{R}\left(,\left(Z_{\mathscr{S}}, Y_{\mathscr{G}}, h\right)\right)\right|_{R_{\mathscr{F}}^{\mathscr{S}}} \rightarrow \operatorname{Hom}_{R}(,(X, Y, g))\right|_{R_{\mathscr{G}}^{\mathscr{S}} \rightarrow 0}
$$

is exact.

To verify this claim, let $(A, B, f)$ be a module in $\bmod R_{\mathscr{T}}^{\mathscr{S}}$ and let $\phi=$ $(\alpha, \beta):(A, B, f) \rightarrow(X, Y, g)$ be an $R$-homomorphism. By the construction of the morphism $t_{Y}: Y_{\mathscr{G}} \rightarrow Y$, there exists a $\Gamma$-homomorphism $\gamma: B \rightarrow Y_{\mathscr{T}}$ such that $\beta$ is the composition $B \stackrel{\gamma}{\rightarrow} Y_{\mathscr{g}} \stackrel{t_{Y}}{\rightarrow} Y$. We then obtain the commuting diagram

$$
\begin{array}{ccccc}
A & = & A & \stackrel{\alpha}{\longrightarrow} & X \\
\downarrow f & & \downarrow \operatorname{Hom}_{\Gamma}(M, \gamma) f & & \downarrow g \\
\operatorname{Hom}_{\Gamma}(M, B) \stackrel{\operatorname{Hom}_{\Gamma}(M, \gamma)}{\longrightarrow} & \operatorname{Hom}_{\Gamma}\left(M, Y_{\mathscr{g}}\right) \stackrel{\operatorname{Hom}_{\Gamma}\left(M, t_{Y}\right)}{\longrightarrow} & & & \\
\operatorname{Hom}_{\Gamma}(M, Y) .
\end{array}
$$

From the property of the pullback

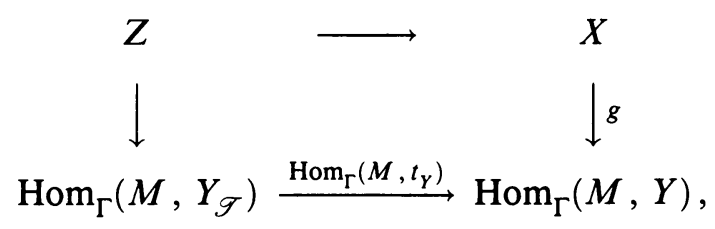

there exists a $\Lambda$-homomorphism $\delta: A \rightarrow Z$ such that

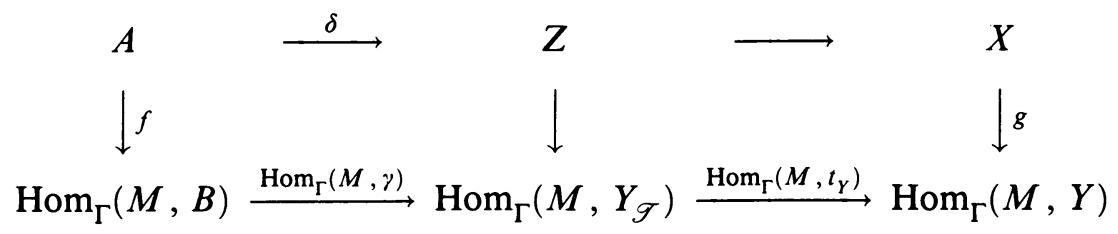

commutes and such that $\alpha$ is equal to the composed homomorphism $A \stackrel{\delta}{\rightarrow}$ $Z \rightarrow X$. By construction of $s_{Z}: Z_{\mathscr{S}} \rightarrow Z$, there exists a $\Lambda$-homomorphism 
$\eta: A \rightarrow Z_{\mathscr{S}}$ such that the following diagram commutes:

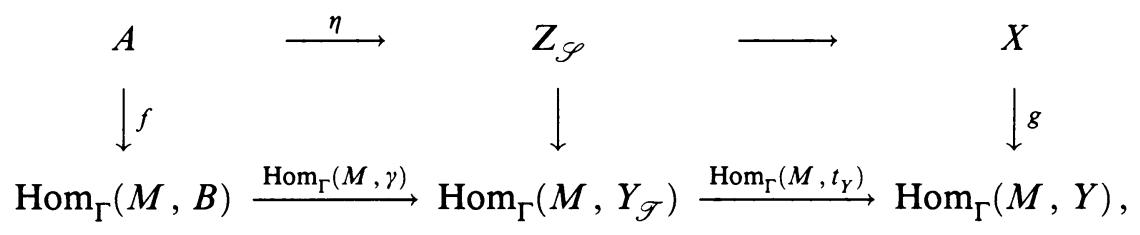

where the composition of the $\Lambda$-homomorphisms in the top row is $\alpha$ and the composition of $\gamma$ and $t_{Y}$ is still $\beta$. This completes the proof of the claim as well as the proof of the theorem.

As an immediate corollary of this result we obtain the following:

Corollary 2.2. If $\mathscr{S}$ is functorially finite in $\bmod \Lambda, \mathscr{T}$ is functorially finite in $\bmod \Gamma$, and both $\mathscr{S}$ and $\mathscr{T}$ are closed with respect to extensions, then $\bmod R_{\mathscr{T}}^{\mathscr{S}}$ has almost split sequences.

Proof. When both $\mathscr{T}$ and $\mathscr{S}$ are closed with respect to extensions, then this also obviously holds for $\bmod R_{\mathscr{G}}^{\mathscr{S}}$. Theorem 2.4 of [AS2] states that a functorially finite subcategory of $\bmod S$ for an Artin algebra $S$ which is closed with respect to extensions has almost split sequences; hence the result follows from the Theorem.

Every full subcategory of $\bmod S$ with only a finite number of isomorphism classes of indecomposable modules is functorially finite in $\bmod S$, when $S$ is an Artin algebra. Therefore, letting $\mathscr{P}$ be the category of projective modules in $\bmod \Lambda$ and letting $\mathscr{I}$ be the category of injective modules in $\bmod \Gamma$, which are both closed with respect to extensions, we immediately obtain that the category $\bmod R_{\mathscr{I}}^{\mathscr{P}}$ has almost split sequences. This is the category of prinjective modules studied by Grecht [Gr] and de la Peña and Simson [PS].

In a forthcoming joint paper with $\mathrm{K}$. Igusa and G. Todorov [IST], the category of modules of finite projective dimension over an Artin algebra will be considered. More precisely, let $S$ be an Artin algebra and let $f p d \bmod S$ be the full subcategory of $\bmod S$ consisting of the modules of finite projective dimension. Some classes of algebras where $f p d \bmod S$ is contravariantly finite will be given, and an example will be constructed showing that $f p d \bmod S$ is not always contravariantly finite in $\bmod S$. Using Theorem 2.1 , we can easily extend the classes of algebras where $f p d \bmod S$ are contravariantly finite in $\bmod S$.

Proposition 2.3. Let $\Lambda, \Gamma, \Gamma M_{\Lambda}$ and $R$ be as before and assume in addition that ${ }_{\Gamma} M$ has finite projective dimension. Then $f p d \bmod R$ is contravariantly finite in $\bmod R$ if and only if $f p d \bmod \Lambda$ is contravariantly finite in $\bmod \Lambda$ and $f p d \bmod \Gamma$ is contravariantly finite in $\bmod \Gamma$.

Proof. From the next lemma we have that $f p d \bmod R$ is the category $\bmod R_{\mathscr{T}}^{\mathscr{S}}$ where $\mathscr{S}$ is $f p d \bmod \Lambda$ and $\mathscr{T}$ is $f p d \bmod \Gamma$. Hence, the result follows directly from the theorem. 
The content of the following lemma may be deduced from results in [FGR], but for the convenience of the reader we include a proof:

Lemma 2.4. Let $\Lambda, \Gamma, \Gamma_{\Gamma} M_{\Lambda}$, and $R$ be as before, and assume that ${ }_{\Gamma} M$ has finite projective dimension. Then $f p d \bmod R$ is $\bmod R_{\mathscr{G}}^{\mathscr{S}}$, where $\mathscr{S}$ is fpd $\bmod \Lambda$, and $\mathscr{T}$ is $f p d \bmod \Gamma$.

Proof. The indecomposable projective $R$-modules are either of form $(P, M \otimes P$, $i d)$ where $P$ is an indecomposable projective $\Lambda$-module or of form $(0, Q, 0)$ where $Q$ is an indecomposable projective $\Gamma$-module. Let $(A, B, f)$ be an $R$-module of finite projective dimension and let

$$
0 \rightarrow\left(X_{n}, Y_{n}, g_{n}\right) \rightarrow \cdots \rightarrow\left(X_{1}, Y_{1}, g_{1}\right) \rightarrow\left(X_{0}, Y_{0}, g_{0}\right) \rightarrow(A, B, f) \rightarrow 0
$$

be a projective resolutior of $(A, B, f)$. Then

$$
0 \rightarrow X_{n} \rightarrow X_{n-1} \rightarrow \cdots \rightarrow X_{1} \rightarrow X_{0} \rightarrow A \rightarrow 0
$$

is a projective resolution of $A$ as a $\Lambda$-module and, using the assumption that ${ }_{\Gamma} M$ has finite projective dimension, we obtain that

$$
0 \rightarrow Y_{n} \rightarrow Y_{n-1} \rightarrow \cdots \rightarrow Y_{1} \rightarrow Y_{0} \rightarrow B \rightarrow 0
$$

is a resolution of $B$ in terms of $\Gamma$-modules of finite projective dimension. Hence, $A$ is in $f p d \bmod \Lambda$ and $B$ is in $f p d \bmod \Gamma$.

Conversely, using induction on the projective dimension on $A$, we obtain that if $(A, B, f)$ is in $\bmod R_{\mathscr{S}}^{\mathscr{S}}$ then $(A, B, f)$ is in $f p d \bmod R$.

\section{REFERENCES}

[AS1] M. Auslander and S. O. Smalø, Preprojective modules over Artin algebras, J. Algebra 66 (1980), 61-122.

[AS2] _ _ Almost split sequences in subcategories, J. Algebra 96 (1981), 426-454.

[FGR] R. M. Fossum, P. A. Griffith and I. Reiten, Trivial extensions of Abelian categories, Lecture Notes in Math., vol. 456, Springer-Verlag, 1975.

[IST] K. Igusa, S. O. Smalø, and G. Todorov, Finite projectivity and contravariant finiteness, Proc. Amer. Math. Soc 109 (1990), 937-941.

[PS] J. A. de la Peña and D. Simson, Prinjective modules, reflection functors, quadratic forms and Auslander-Reiten sequences, preprint, 1989.

[Gr] R. Grecht, Kategorien von Moduln mit Untermoduln, Diplomarbeit, Zürich, 1986.

Institutt for Matematikk og Statistikk, Universitetet i Trondheim, AVH, N-7055 DRAGVOLL, NORWAY 